\title{
Offline impairment-aware RWA and regenerator placement in translucent optical networks
}

\author{
B. Garcia-Manrubia, P. Pavon-Marino, R. Aparicio-Pardo, M. Klinkowski and D. Careglio
}

\begin{abstract}
In translucent optical networks the physical layer impairments degrading the optical signal are considered in the network design. In this paper we investigate the offline problem of Routing and Wavelength Assignment (RWA) and Regenerator Placement (RP) in translucent networks. Given a network topology, an estimation of the traffic demands, the objective is to minimize the cost of the regeneration equipment used, and to avoid the lightpath blocking. We formulate an optimal ILP model of the problem, to the best of the authors' knowledge, for the first time in the literature. Its simplicity allows us to test it for small and medium size networks. Despite of this merit, the problem is NP-hard. For larger problem instances we propose two heuristic methods: Lightpath Segmentation and 3-Step method. The latter guarantees that no lightpath blocking is produced by signal degradation. We also provide a lower bound for the regenerator equipment cost. The performance and the scalability of our proposals are then investigated by carrying out extensive tests, considering different network topologies, number of wavelengths per fiber, traffic load conditions and network link lengths. Results reveal that the solutions obtained by the heuristic algorithms are optimal or close-to-optimal and require low computation times. In addition, the results help to capture the trends in the regenerator equipment cost in different network instances.
\end{abstract}

Index Terms-Impairment Aware Network Planning, Regenerator Placement, Translucent Optical Networks.

\section{INTRODUCTION}

$\mathrm{T}$ HE popularization of bandwidth-hungry applications and services is enforcing the deployment of optical WDM networks with a clear trend towards increasing the capacity and lowering the network cost (both CAPEX and OPEX). On

The work described in this paper was carried out with the support of the BONE project ("Building the Future Optical Network in Europe"); a Network of Excellence funded by the European Commission through the 7th ICTFramework Program. This research has been partially supported by the MEC project TEC2007-67966-01/TCM CON-PARTE-1, it is also developed in the framework of the projects from Fundación Seneca 00002/CS/08 (FORMA) and "Programa de Ayudas a Grupos de Excelencia de la Región de Murcia", F. Séneca (Plan Regional de Ciencia y Tecnología 2007/2010)", and a research project supported by the Polish Ministry of Science and Higher Education.

B. Garcia-Manrubia, P. Pavon-Marino, and R. Aparicio-Pardo are with Technical University of Cartagena (UPCT), 30202 Cartagena, Spain (e-mail: \{belen.garcia, pablo.pavon, ramon.aparicio\}@upct.es).

M. Klinkowski is with the National Institute of Telecommunications, Poland (e-mail: mklinkow@itl.waw.pl).

D. Careglio is with the Technical University of Catalonia, Spain (e-mail: careglio@ac.upc.edu). the one hand, this trend can be translated to higher, 40/100 Gbps line rates and denser WDM transmission systems with 80 to 160 wavelengths per fiber. On the other hand, aiming at reducing the cost, recent advances in optical technologies are fostering an evolution from traditional opaque to transparent optical network architectures [1].

In an opaque network, each node is equipped with Optical Electrical Optical (OEO) interfaces meaning that the optical signal carrying traffic terminates at each node to undergo an OEO conversion and an electronic processing. This approach simplifies the network design and control since there is a full independence between the network and the physical layer. On the contrary, it requires a large amount of OEO devices greatly increasing the network cost and the energy consumption.

In transparent optical network, the optical signal originated at the source nodes reaches its destination bypassing optically the intermediate nodes. This approach reduces considerably the cost since neither OEO conversions nor electronic processing is required at each node along a lightpath. However, it implies that the physical layer must support endto-end communication. Unluckily, the transmission reach of optical signals is limited due to the accumulation of physical layer impairments which cause signals' quality of transmission (QoT) - for example the Bit Error Rate (BER) to drop beyond an acceptable level.

For that very reason, translucent (or semi-transparent) optical networks are emerging as a promising solution for bridging the gap between opaque and transparent networks. Indeed, translucent networks combine features of both opaque and transparent networks strategically placing electrical regeneration (i.e. by means of OEO conversion) only at selected points in the network [2]. This approach eliminates much of the required electronic processing and allows a signal to remain in the optical domain for much of its path. Moreover, an electrical regenerator enables the possibility of wavelength conversion which may help to decrease the number of rejected lightpaths compared to the transparent case.

In both transparent and translucent optical networks, the network and the physical layer cannot be decoupled (as in opaque networks) and a cross-layer design is necessary. The traditional problem of routing and wavelength assignment (RWA) must take into account the signal QoT in the lightpath computation process in the so called impairment aware RWA (IA-RWA). Two cases can be faced. During the planning 
phase, the traffic demand is already known at least partially; therefore the decisions can be taken offline using static IARWA algorithms. The other case, whereby traffic demands are assumed to arrive in a dynamic fashion, is referred to as the online or dynamic IA-RWA problem. A comprehensive literature review of proposed static and dynamic IA-RWA algorithms can be found in [3].

Besides, in the translucent optical networks, there are the additional problems of regenerator placement and allocation. In the planning phase, the regenerator placement consists of selecting which nodes of the network have regeneration capabilities and how many signals can be regenerated at these nodes. In contrast, in the operation phase, the regenerator allocation tries to determine how the already placed regenerators are used in a dynamic scenario.

In all these issues, a QoT estimator accounting for the accumulation of the physical layer impairments along the path and thus determining the signal QoT is needed. These impairments include chromatic and polarization-mode dispersion, optical-fiber nonlinearities, noise accumulated due to amplified spontaneous emission (ASE); cross-talk; etc. In literature, there are three main QoT estimators based on the numerical calculation of the Optical Signal-Noise Ratio (OSNR) [4], applying analytical formulas [5] or interpolating numerical and laboratory measurements [6], [7] to compute the $Q$ factor value.

In this paper we focus on translucent optical networks and we address the offline problem of network planning where, given a network topology and an estimation of the traffic demands, both the static IA-RWA and the regenerator placement problems are solved jointly. We denote this problem as IA-RWA-RP (IA-RWA-Regenerator Placement). As a QoT estimator, we use the extended Q-Personick model defined in [7]. Our target is to find the network design which eliminates the lightpath blocking caused by the optical signal degradation and wavelength conversion requirements, minimizing the cost of the regeneration equipment. We denote the lightpath blocking directly caused by the signal degradation and wavelength conversion requirements as signal regeneration blocking. Note that in the offline planning of translucent networks there is other source of lightpath blocking: the one caused by the limited number of wavelengths of the links. We name this second type of blocking as network capacity blocking. The network capacity blocking cannot be solved by using more regenerator equipment: if no routes with available channels exist between two nodes, no wavelength conversion or regenerator equipment will prevent a lightpath between those nodes to be blocked.

In this paper, we model the IA-RWA-RP problem as an ILP (Integer Linear Programming) combining a node-link formulation with the concept of semi-lightpath. A semilightpath is an optical signal traversing a sequence of fiber links without going through any signal regeneration and any wavelength conversion. Then, a lightpath is implemented as a chain of semi-lightpaths, requiring one regenerator where each semi-lightpath ends (but the last). We emphasize as a merit of this model its simplicity. Thanks to it, it has been possible to obtain optimal solutions for the IA-RWA-RP problem in medium size networks like Internet2 an NSFNET with 16 wavelengths per fiber. For planning larger networks, we present two effective heuristic algorithms: the Lightpath Segmentation (LS) and the 3-Step heuristics, together with a lower bound to the regenerator equipment cost which is measured in total number of regenerators used. The 3-Step heuristic is designed to guarantee a 0 signal regeneration blocking.

An extensive battery of tests is included in this paper. The tests have been carefully selected, so that they correspond to networks which allow solutions with a 0 network capacity blocking. Then, we are able to fairly assess both the signal regeneration blocking and the regenerator cost of the solutions provided. As a contribution of this paper, we also investigate how the regeneration equipment cost increases for different network sizes. For this, we define a normalized network size factor, which captures the relative length of the network links with respect to the longest signal propagation without regeneration.

The rest of the paper is organized as follows. In Section II we review the related work. Section III presents an ILP formulation to solve optimally the problem proposed. In Section IV we provide the description of two heuristic algorithms proposed. Section V describes the conducted experiments and the obtained results. Finally, Section VI concludes the paper.

\section{RELATED WORK}

The first studies on the IA-RWA problem for translucent optical networks propose to divide the optical core network into several islands of transparency or optically transparent domains [8], [9]. An island consists of a part of the physical topology in which any lightpath can be established without intermediate signal regeneration. If a connection traverses several islands, the island boundary nodes carry out the signal regeneration. The same idea has been employed in some recent works such as [10]. The problem of this approach is its low scalability. Only the nodes on the islands' borders can host a regenerator and, in order to minimize the number of regenerators, these islands must be defined ensuring a minimum overlapping. Any change in the network (a failure, an upgrade, etc.) may require a reorganization of the islands and a re-placement of the majority of the regenerators.

An alternative approach called sparse regenerator placement is being studied in [11]-[24]. In this case any node can host in principle a regenerator (not only the nodes on the islands' border) and a regenerator placement algorithm defines the subset of nodes that actually need a regenerator: in the end they are deployed sparsely in the network. This is also the approach considered in this paper. 
The majority of the proposals for regenerator placement in translucent networks deal with the IA-RWA and the regenerator placements problems separately, as if they were two different phases of the network design. Commonly, the regenerator placement is solved first, producing a network for which the IA-RWA problem is then addressed. This strategy has been followed in [14]-[18] for static traffic, and in [11][13], [19]-[21] considering dynamic traffic. Other works have been presented which focus on minimizing the number of nodes with signal regeneration capability in the network, guaranteeing a certain degree of connectivity [19], [22], [23].

In [12], [13], [21] the authors study the regenerator placement and the regenerator allocation problem. The former plans the number of regenerators to be placed in each node assuming a non-deterministic traffic demand. The latter, allocates the idle regenerators to the dynamic arrivals of new connection requests. Four static algorithms for the sparse regenerator placement and two dynamic schemes for the regenerator allocation are proposed. The regenerator placements are based on static network states and merely empirical considerations: either placing the regenerators considering the maximum transmission reach of a transparent path, in the most central nodes, in the nodes with the higher number of links, or in those with the higher loads. The regenerator allocations called fragmentation and trace back reorganize how the regenerator equipment in each node is assigned taking into account the current network state. The placement-allocation process is investigated also in [20]. A heuristic algorithm is firstly applied for the regenerator placement; the nodes crossed by the majority of the shortestpaths host the regenerators. Then, the IA-RWA and the regenerator allocation problems assuming dynamic traffic are dealt with a two-dimensional Dijkstra algorithm.

In [15] a MILP formulation and a sequential heuristic algorithm based on the $K$-least-wavelength-weight-path routing are proposed to solve the IA-RWA problem. The objective of both schemes is the maximization of the number of established connections which means that a connection blocking can occur. In principle, any node can host a regenerator but the total number of regenerators is an input value and it is not minimized.

In [22] the problem studied consists of minimizing the number of nodes with signal regeneration capability guaranteeing a desired degree of end-to-end connectivity in the optical network. To this end, they suggest a two-step algorithm which is compared to a $k$-coverage algorithm for mobile and ad hoc wireless networks. The authors in [19], [23] propose a regenerator placement based on the greedy algorithm from [24] to get full-connectivity and two simple RWA algorithms based on shortest path and assigning the wavelengths by first-fit. One model considers the worst-case physical transmission penalties, while the other model takes into account the current network status in order to obtain the physical impairments.

In contrast to previous works, in this paper we study how to solve the regenerator placement and the IA-RWA problems jointly which have been previously studied only in [14] and [18].

In [14] a sequential algorithm called LERP (Lightpath Establishment with Regenerator Placement) is proposed. This algorithm minimizes simultaneously the number of rejected traffic demands and the number of required regenerators. In [16], [17] the authors continue their study. They investigate the impact of deploying in-line gain equalizers in terms of the number of required regenerators. Moreover, they propose two new wavelength assignment strategies to employ in the LERP algorithm.

In [18] the offline IA-RWA-RP problem is divided into three phases. Given a set of lightpaths demands (i.e. the traffic matrix), the first phase finds those lightpaths that cannot be served transparently by any of the $k$-shortest paths available. An ILP model and four different heuristic algorithms are proposed to decide how to split all these non-transparent lightpaths into a sequence of transparent lightpaths and place the regenerators accordingly. The result of this phase is a transformed traffic matrix. In the second phase, an IA-RWA algorithm is applied to route the transformed traffic matrix. If some connections cannot be served, a third phase tries to reroute them using the remaining network resources. However, at the end of all these phases, some connections can be still blocked due to unacceptable QoT performance.

In this article we propose a novel ILP formulation, yielding optimal solutions to the problem and being the first one to achieve this objective as far as the authors know. The simplicity of the model allows to optimally solving small/medium scale non-trivial problems. For larger networks we also present a regenerator cost lower bound, and two novel heuristics. These heuristics have shown very good scalability properties, and accurate results, outperforming previous proposals.

\section{PROBLEM FormULATION}

In this section we present an exact ILP formulation which solves optimally the IA-RWA-RP problem. The input parameters are the physical topology, the lightpath demands and the physical impairments. Let $N$ be the set of nodes in the network, $E$ the set of unidirectional fiber links, and $W$ the set of wavelengths in each link. We assume that all fibers in the network have the same number of wavelengths. We denote as $a(e)$ and $b(e)$ the initial and ending nodes of fiber $e \in E$. We also denote $\delta^{+}(n)$ and $\delta^{-}(n)$ the set of fibers initiated and ending at node $n \in N$ respectively. $T$ denotes the set of lightpath demands, being $T_{s d}$, the number of lightpaths to be established from node $s$ to $d, s, d \in N$.

Our formulation is based on the concept of semi-lightpath. As a pre-processing step, the set of valid semi-lightpaths $P_{Q}$ is calculated in the network. They consist of the set of all the paths which are valid considering the physical impairments. In the results section, we apply the Q-Personick [25] estimator as a measure for the degradation produced by the physical impairments. Naturally, the model proposed can be applied 
with other QoT estimators of the signal impairments.

The model presented in this paper uses the semi-lightpaths as the links in a node-link formulation of the problem. We assume that regenerators are capable of wavelength conversion. The decision variables of the problem are:

- $x_{p w s d}=\{0,1\}, p \in P_{Q}, w \in W, s, d \in N . x_{p w s d}$ takes the value 1 if the path $p$ in $P_{Q}$ uses the wavelength $w$ for carrying one lightpath of the demand from $s$ to $d$.

$-t_{s d}=\{0,1,2, \ldots\}, s, d \in N$. Number of lightpaths carried from node $s$ to node $d$.

The problem formulation is given by (1):

$$
\min \left(\sum_{p \in P_{Q}, w \in W, s, d \in N} x_{p w s d}-\sum_{s, d \in N} t_{s d}\right)+M \cdot\left(\sum_{s, d \in N}\left(T_{s d}-t_{s d}\right)\right)
$$

subject to:

$$
\begin{aligned}
& \sum_{\substack{p \in \delta^{+}(n) \\
w \in W}} x_{p w s d}-\sum_{\substack{p \in \delta^{-}(n) \\
w \in W}} x_{p w s d}=\left\{\begin{array}{l}
t_{s d} \text { if } n=s \\
-t_{s d} \text { if } n=d, \forall n, s, d \in N \\
0 \text { otherwise }
\end{array}\right. \\
& \sum_{\substack{p \in P_{Q e}, s, d \in N \\
t_{s w s d}}} \leq 1, \forall e \in E, w \in W \\
& t_{s d} \leq T_{s d}, s, d \in N
\end{aligned}
$$

The objective function (1a) consists of two elements. The first component aims to minimize the number of regenerators to be placed. The number of optical regenerators required by a lightpath is given by its number of semi-lightpaths minus 1 . Then, the total number of regenerators is given by the total number of active semi-lightpaths, minus the number of carried lightpaths. The second part of the objective function deals with the minimization of the number of blocked lightpath requests. By using a constant $M$ high enough it is possible to set the blocking minimization as the dominating criteria in the optimization (e.g. $M=|W||N|)$. Then, a solution which carries more traffic would be always preferred whatever amount of extra signal regenerators requires. Constraints (1b) are the flow conservation constraints for the link-flow formulation. Constraints (1c) avoid the wavelength clashing, i.e., a wavelength in a fiber can be used only once. We denote as $P_{Q e}$ to the set of semi-lightpaths which traverse the fiber $e$. Finally, constraints (1d) state that carried traffic is limited by the offered traffic.

Note that this model can be easily modified adding a placement constraint; in such a case, only a subset $N_{r}$ of the nodes is allowed to be equipped with regenerator/converter devices. The constraint (1e) introduces this into the model:

$x_{p w s d}=0, \forall p \in P_{Q}, b(p) \neq d, w \in W, s \in N, d \notin N_{r}$

In (1e), the semi-lightpaths ending in a node without regenerator equipment capability can only be active if they are the last semi-lightpath of the lightpath. That is, for a semilightpath $p$, its ending node, which we denote as $b(p)$, does not need to belong to the subset $N_{r}$ when $b(p)$ is the ending node of the lightpath.

\section{HEURISTICS}

The IA-RWA-RP problem is clearly NP-hard, since it contains the RWA NP-hard problem [26] as a special case. Herein, we propose two simple heuristic algorithms with the objective to solve the IA-RWA-RP problem.

\section{A. Lightpath Segmentation Algorithm}

A lightpath segmentation (LS) algorithm is an ILP-based algorithm which applies the concept of semi-lightpaths introduced in Section I. On the contrary to the ILP formulation (OptILP) presented in Section III, which makes use of pre-computed semi-lightpaths that are composed into lightpaths, the idea behind LS is to start with pre-defined endto-end paths which are then decomposed onto transparent semi-lightpaths. Besides, we must highlight that OptILP considers the set of all the existing valid semi-lightpaths obtaining an optimal solution, while LS calculates a reduced set of candidate paths to limit the complexity of the formulation and, thus, a heuristic solution is found. To formulate the problem we use a similar notation as in Section III.

We assume that each lightpath that is established in the network follows an explicit routing path. Accordingly, as a pre-processing step, a set of candidate paths $L_{s d}$ (e.g., $k$ shortest paths) is calculated for each pair of nodes $s, d \in N, s \neq d$. Let $L$ denote the set of all paths.

Let $L^{\text {reg }}$ and $L^{\text {noreg }}$ denote, respectively, a subset of paths requiring regeneration at some intermediate node(s) and a subset of paths with no regeneration required; $L=L^{\text {reg }} \cup L^{\text {noreg }}$. For paths $p \in L^{r e g}$, which are not valid considering the physical impairments, a segmentation procedure is performed in the pre-processing step to decompose them on valid semilightpaths. We perform such a procedure iteratively by segmenting a path on a number of sub-paths and by checking the physical impairment validity of each sub-path. Since path $p$ may be segmented in a number of ways, we obtain a set of candidate segmentations $R_{p}$. Each segmentation corresponds to a sequence of valid semi-lightpaths composing path $p$. In order to limit the size of $R_{p}$, in our implementation of the segmentation procedure, we begin the search with the lowest number of sub-paths (i.e., two) that divides a given path and increment their number until a valid segmentation is found.

Having calculated $L$ and $R_{p}$, where $p \in L^{r e g}$, in the preprocessing step, the LS problem concerns the selection of path $p$ from the set of candidate paths $L$ for each lightpath request and, if $p \in L^{r e g}$, the selection of a segmentation of this lightpath from the set of candidate segmentations $R_{p}$. Concurrently, the RWA constraints, such as wavelength continuity, flow conservation, wavelength capacity, etc., have to be satisfied 
for all established lightpaths in the network. We continue formulating the optimization problem. First, the set of problem coefficients and constants coming from the preprocessing step are as follows:

- $l_{p r}, p \in L, r \in R_{p}$. Number of transparent segments (semilightpaths) on path $p$ in $L$ under segmentation $r$ in $R_{p}$. - $\delta_{e p}, e \in E, p \in L$. Coefficient which is equal to 1 if link $e$ belongs to path $p$, and equal to 0 otherwise.

- $\eta_{\text {elpr }}, e \in E, l \in\left\{1, \ldots, l_{p r}\right\}, p \in L^{r e g}, r \in R_{p}$. Coefficient which is equal to 1 if link $e$ belongs to segment $l$ of path $p$ under segmentation $r$, and equal to 0 otherwise.

- $M$. Big constant number used as a weighting coefficient to give a priority to the blocking objective over the regenerator usage objective; to achieve it, it is enough to have $M=\left|L^{r e g}\right| \cdot|W| \cdot\left(\max \left\{s_{p r}: p \in L^{r e g}, r \in R_{p}\right\}-1\right)+1$.

The problem decision variables are the following:

- $x_{s d} \in Z_{+}, \quad s, d \in N$. Number of not-accepted lightpath requests from node $s$ to node $d$.

- $x_{p} \in Z_{+}, p \in L$. Number of accepted lightpath requests that follow path $p$.

- $x_{p w} \in\{0,1\}, p \in L, w \in W . x_{p w}$ is equal to 1 if wavelength $w$ on path $p$ is assigned to a lightpath (in case if $p \in L^{\text {noreg }}$ ) or to the first semi-lightpath (if $p \in L^{\text {reg }}$ ), and equal to 0 otherwise.

- $x_{p w r l} \in\{0,1\}, p \in L, r \in R_{p}, w \in W, l \in\left\{1, \ldots, l_{p r}\right\} . x_{p w r l}$ is equal to 1 if semi-lightpath $l$ on path $p$ under candidate segmentation $r$ has assigned wavelength $w$, and equal to 0 otherwise.

The formulation is described in (2):

$\operatorname{minimize} \sum_{p \in L^{\text {reg }}} \sum_{w \in W} \sum_{r \in R_{p}}\left(l_{p r}-1\right) x_{p w r 1}+M \sum_{s, d \in N} x_{s d}$

subject to:

$\sum_{p \in L_{s d}} x_{p}+x_{s d}=T_{s d}, \quad \forall s, d \in N$

$\sum_{w \in W} x_{p w}=x_{p}, \quad \forall p \in L$

$\sum_{r \in R_{p}} x_{p w r 1}=x_{p w}, \quad \forall p \in L^{r e g}, w \in W$

$\sum_{w \in W} x_{p w r(l-1)}=\sum_{w \in W} x_{p w r l}, \quad \forall p \in L^{r e g}, r \in R_{p}, l=2 \ldots l_{p r}$

$\sum_{p \in L^{\text {noreg }}} \delta_{e p} x_{p w}+\sum_{p \in L^{\text {reg }}} \sum_{r \in R_{p}} \sum_{l=1 \ldots l_{p r}} \eta_{e l p r} x_{p w r l} \leq 1, \quad \forall e \in E, w \in W$

The objective function (2a) consists of two components. The first one counts the number of regenerators which depends on the number of semi-lightpaths that compose the lightpaths requiring signal regeneration. The second component represents the number of blocked lightpath requests. The constant $M$ is assumed to be big enough so that the blocking objective dominates the optimization. Constraints (2b) are the traffic constraints, which mean that either the offered connection requests are distributed over candidate paths or they are lost. Constraints (2c) and (2d) are the wavelength assignment constraints both for the lightpaths that do not require the signal regeneration and for the lightpaths that are composed of semi-lightpaths. In particular, in (2d) the wavelength of the first semi-lightpath together with a convenient segmentation of the corresponding lightpath is determined. Concurrently, constraints (2e) are the flow conservation constraints which assign wavelengths to consecutive semi-lightpaths under the assumption that the regenerative nodes are capable of the wavelength conversion. Eventually, constraints (2f) represent the wavelength capacity constraints.

Since optimization problem (2) is a variant of the RWA problem (to see it, consider $L^{\text {reg }}=\varnothing$ ), the problem is NP-hard. Nevertheless, under the condition that the sets of candidate paths and candidate segmentations are not large, the algorithm performance is satisfying, even for larger network instances, as shown in the results section.

\section{B. 3-Step Heuristic}

The second heuristic algorithm proposed consists of three consecutive steps.

Step 1 (Routing): An instance of the Integral Multicommodity Flow Problem [27] is optimally solved for the network. In the Integral Multicommodity Flow formulation, each link represents a fiber in the original network, with a capacity given by $|W|$. The flows to allocate are the lightpaths. Each carried lightpath occupies a capacity of one in each traversed fiber. The purpose of this formulation is to find a route for every lightpath demand without considering either the wavelength assignment or the physical impairments. The objective function is set to (i) minimize the lightpaths blocked, and (ii) among the solutions with the minimum blocking, search for the one minimizing the average number of physibal hops of the carried lightpaths.

Step 2 (Wavelength assignment and converter placement): The carried lightpath 2 cfrom the previous stage are sequentially processed. For each lightpath, a first fit wavelengthd) assignment is carried out. When wavelength continuity is not possible, regenerators are used as wavelength converters. We use the first wavelength which allows carrying the lightpath using one regenerator. If that is not possible, the same is applied for 2, 3 etc. regenerators, till a solution is found.

Step 3 (Regenerator placement): The set of lightpaths produced from previous iterations are sequentially processed. The $Q$ factor of each lightpath is evaluated. If its $Q$ factor value is below the QoT threshold set, it is trivially split into the minimum number semi- 
lightpaths needed, placing the regenerators in the appropriate nodes.

Step 1 of the algorithm, produces the set of lightpaths to be carried, minimizing the network capacity blocking. After that, Step 2 and 3 of the algorithm heuristically search for the minimum number of regenerators which solve the wavelength clashing and signal degradation issues. However, note that no blocking exists in both last steps. Therefore, the 3-Step Heuristic optimally minimizes the network capacity blocking, and guarantees a 0 signal regenerator blocking.

Finally, while the integral version of the multicommodityflow problem used in Step 1 is known to be NP-hard, its complexity has shown to be acceptable for the network sizes of interest, as shown in the results section.

\section{RESUlts}

This section collects and analyzes extensive results obtained for validating the algorithms proposed, under different testing scenarios. As a comparison, we also provide the lightpath blocking and regenerator cost performances calculated by the LERP algorithm proposed in [14]. All the algorithms have been implemented in MATLAB code, integrated and tested in the MatPlanWDM tool [28], which interfaces with the TOMLAB/CPLEX solver [29].

\section{A. Testing scenarios}

Three reference network topologies, together with their corresponding reference traffic matrices are used in our study: Internet2 [30], NSFNET [31], and NOBEL-EU [32]. Table I summarizes some major data from these topologies. All the nodes are allowed to host regenerators. The number of wavelengths per fiber tested is $W \in\{8,16\}$ for medium-sized networks Internet 2 and NSFNET, and $W \in\{80\}$ for the NOBEL-EU test.

In this paper, we assume a physical layer impairment model based on the so-called Personick's $Q$ factor [25]. The $Q$ factor estimates the quality of optical signal along a transparent semi-lightpath, i.e., the segment of a lightpath comprised between two regenerators. The Personick's $Q$ factor takes into account both linear and non-linear effects. The measure of linear effects in the $Q$ factor computation is the Optical Signal-to-Noise Ratio (OSNR). The semi-lightpath OSNR can be calculated considering the OSNR across each of the elementary optical system components (such as the fiber spans and the nodes) along the semi-lightpath and by combining the partial results. The non-linear effects are incorporated into the model by means of some empirical signal degradation factors counted for all amplifiers (both boosters and in-line amplifiers) along the semi-lightpath. For more details on the $Q$ factor calculation we refer to [7].

The optical transmission system parameters considered in the evaluation scenario are the same as in [7]. In particular, we assume a transmitter bit rate of $10 \mathrm{Gbps}$ and spans of standard single mode fiber (SMF). The span length of the fibers is set to $85 \mathrm{~km}$. The fiber attenuation considered is $0.23 \mathrm{~dB} / \mathrm{km}$, with an additional margin of $3 \mathrm{~dB}$ per span. Other parameters used to characterize the physical layer are the noise figure of in-line amplifiers and pre-amplifiers $(5 \mathrm{~dB})$, the noise figure of boosters $(6 \mathrm{~dB})$, switching power attenuation $(13 \mathrm{~dB})$, and the signal launch power at the input of the fiber spans (3 $\mathrm{dBm})$. The threshold on the acceptable $Q$ factor value is equal to $17 \mathrm{~dB}$.

It is worth noting that the proposed network planning methods can be used with any physical layer impairment estimator as far as the estimator accounts for static impairments, i.e., the ones that do not vary in time, and dynamic impairments are represented by means of a (constant) worst-case signal degradation component. This is the case of the Q-Personick estimator we use in this paper. Under such assumption, the role of the estimator is only to validate the semi-lightpaths found in the pre-processing phase of the algorithms.

In our tests, three traffic loads are considered: low, medium and high $(\rho \in\{0.4,0.7,1\})$. Given a network topology, a reference traffic matrix for that topology $T^{B A S E}$ (measured in any arbitrary traffic units), a number of wavelengths per fiber $W$ and a traffic load factor $\rho$, the lightpath demand matrix is calculated as follows. First, we calculate the maximum lightpath demand matrix $T^{M A X}$. This is done by finding the maximum value $\alpha^{M A X}$ for which the lightpath demand matrix in (3) admits a feasible routing solution for an instance of the integral multicommodity flow problem (built as in the Step 1 of the 3-Step algorithm). This guarantees that the lightpaths in $T^{M A X}$ can be fully carried by the network with a $0 \%$ of lightpath blocking if a sufficient number of regenerators are used.

$T^{M A X}=\operatorname{round}\left(\alpha^{M A X} \cdot T^{B A S E}\right)$

The maximum lightpath demand matrix, $T^{M A X}$ is associated to load $\rho=1$. Let us denote $S_{T}$ the total number of lightpaths in $T^{M A X}, S_{T}=\sum_{s, d \in N} T^{M A X}$ sd . Then, the lightpath demand matrices $T^{\rho}$ at other network loads $\rho$, are calculated by finding the factor $\alpha<\alpha^{M A X}$ for which its associated lightpath demand matrix has a volume equal to $\rho S_{T}$ (or its closest integer value).

$T^{\rho}=\left\{\begin{array}{l}\operatorname{round}\left(\alpha \cdot T^{B A S E}\right), \text { so that } \alpha \leq \alpha^{M A X}, \\ \sum_{s, d \in N} T^{\rho}=\operatorname{round}\left(\rho S_{T}\right)\end{array}\right\}$

The traffic normalization designed in our tests implies that all the planning instances admit a solution with a $0 \%$ of network capacity blocking. 
TABLE I

INFORMATION ON THE TOPOLOGIES TESTED

\begin{tabular}{c|ccc}
\hline & Internet2 & NSFNET & $\begin{array}{c}\text { NOBEL- } \\
\text { EU }\end{array}$ \\
\hline Reference & {$[30]$} & {$[31]$} & {$[32]$} \\
Nodes & 9 & 14 & 28 \\
Unidirectional links & 26 & 42 & 82 \\
Average in degree (link) & 1.44 & 1.50 & 1.46 \\
Average link length $\beta=\beta^{M I N}(\mathrm{~km})$ & 531.3 & 432.4 & 292.2 \\
Average link length $\beta=\beta^{M A X}(\mathrm{~km})$ & 1806.4 & 1081 & 1043 \\
Maximum distance without & 2688 & 2688 & 2688 \\
regeneration $(\mathrm{km})$ & & & \\
Ratio maximum/average link & 1.6 & 2.6 & 2.5 \\
\hline length $(>1)$ & & &
\end{tabular}

We are also interested in studying the effects of the network link lengths on the regenerator equipment cost planned. For each network topology, we follow this sequence of steps:

1) We calculate the maximum factor $\beta^{M A X}$ so that after multiplying the length of all the links of the network by $\beta^{M A X}$, the longest link has a $Q$ factor value just equal to the acceptable detection threshold $(17 \mathrm{~dB})$. Note that values $\beta>\beta^{M A X}$ are not considered, since they would require an optical signal to be regenerated in the middle of a fiber link.

2) We calculate the minimum factor $\beta^{M I N}$ so that after multiplying the length of all the links of the network by $\beta^{M I N}$, the shortest path between every pair of nodes with the worse (lowest) $Q$ factor still has a $Q$ factor $Q=17 \mathrm{~dB}$. As will be shown later, distance factors $\beta<\beta^{M I N}$ result in networks which do not require regenerator equipment even at high loads.

3) We repeat the tests for four distance factors in the network: $\left\{\beta^{1}, \beta^{2}, \beta^{3}, \beta^{4}\right\}$, where $\beta^{1}=\beta^{M I N}, \beta^{4}=\beta^{M A X}$, and we obtain $\beta^{2}$ and $\beta^{3}$ as intermediate points between the values of $\beta^{1}$ and $\beta^{4}$.

Note that $\beta$ multipliers define a sort of normalized network size. They depend on the relation between the link lengths and the QoT degradation. This latter effect is affected by technological aspects like the transceivers bit rate and modulation, or the amplification and compensation equipment installed in the network. In other words, higher $\beta$ multipliers could be associated either to continental long-haul networks, or to smaller networks with e.g. shorter reach transmission technologies.

\section{B. Results}

Table II collects the testing results for the networks Internet 2 and NSFNET. In both cases, the tests has been conducted for $W \in\{8,16\}$ wavelengths, three load levels $\rho \in\{0.4,0.7,1\}$ and 4 distance factors $\beta \in\left\{\beta^{1}, \beta^{2}, \beta^{3}, \beta^{4}\right\}$. Four regenerator placement methods are compared: (i) OptILP, the exact formulation presented in Section III, (ii) LS heuristic in Section IV.A, (iii) 3-Step heuristic in Section IV.B, and (iv) the LERP algorithm proposed in [14]. The
LERP algorithm has been executed ranging different values of a set of specific parameters that tune how the solution space is heuristically explored. The results shown in this paper correspond to those parameters which provided the best performances. We reproduce their values to allow the results in this paper to be repeatable: (i) the number of shortest paths computed associated to each demand is set to 4, (ii) the LERP black list size is set to 100 , and (iii) the number of permutations performed in the demand set is 10000 . The reader should refer to [14] for more details on the operation of the LERP algorithm. For the LS algorithm the set of candidate paths is calculated as $k$ shortest paths between every pair of nodes according to the physical distance. When the value of $k$ is selected, we must take into account that using more candidate paths avoids the problem of network capacity blocking, but it increases the algorithm complexity. Therefore, we execute several experiments for each topology scenario and find the lowest number of candidate paths that allows to reach the 0 -blocking objective, in particular, $k=3, k=5$, and $k=2$, respectively, for Internet2, NSFNET, and NOBEL-EU.

In Table II we provide the information related to the regenerator equipment cost and the execution time of the algorithms. The regenerator cost is given as the average number of regenerators that a carried lightpath needs (in \%). That is, the total number of regenerators planned divided by the carried demand volume. The lightpath blocking information is not provided for the OptILP, 3-Step, and LS algorithms since it is 0 for all of them. Note that this was guaranteed for both the OptILP and 3-Step methods. Results have shown that although LS heuristic does not necessarily guarantee a $0 \%$ of signal regeneration lightpath blocking, it provides this benefit in practice thanks to the appropriate choice of the set size of pre-defined paths. In contrast, the LERP algorithm exhibits lightpath blocking in numerous cases.

The column LB provides a lower bound to the regenerator cost. It corresponds to the number of regenerators needed if each of the lightpaths was carried alone in the network using the path with the smallest $Q$ factor, that is, with the lowest number of semi-lightpaths traversed. The interest on plotting the LB information in Table II, is evaluating its accuracy by comparing its value to that of the optimal cost in OptILP column. The accuracy of the LB has shown to be perfect in the Internet2 topology. For the NSFNET network, a null or small gap between the LB and the optimal cost is observed in all the low and medium load occasions. A more significant gap between the LB and the optimal cost is found in high network loads and high normalized network sizes (with a maximum of $\sim 15 \%$ in the worst case). This is logical, since the lower bound assumes a shortest path routing, which is in general not possible at high load conditions.

By comparing the regenerator cost performance in OptILP with those from the heuristic algorithms, we can assess their quality for the small to medium size topologies tested. Results in Table II show that the number of regenerators required by the 3-Step and LS algorithms are equal to the results obtained 
by OptILP in almost all the cases. On the contrary, the LERP algorithm obtains accurate results only for the distance factor $\beta^{1}$. For the rest of scenarios, it needs a higher number of regenerators and/or incurs in lightpath blocking. Note that in some occasions, the regenerator cost of the LERP solution is lower than the optimum. This is because LERP has significant lightpath blocking and, therefore, requires a smaller number of regenerators. In summary, the 3-Step and LS algorithms outperform the LERP algorithm for the scenarios considered obtaining a close to optimal regenerator cost, without incurring in lightpath blocking.

Table III collects the results of the heuristic algorithms for the case of NOBEL-EU network, and $W=80$ wavelengths per fiber. Given the larger number of nodes and wavelengths, it has not been possible to obtain results with OptILP. However, it is very interesting to see that in this case, both the 3-Step and LS heuristics achieve the optimum solution that minimizes the regenerator equipment cost with a $0 \%$ of lightpath blocking. Optimality is guaranteed since its cost equals the cost of the lower bound. The results of 3-Step and LS algorithms outperform the ones of LERP, which incurred in lightpath blocking in the instances with normalized network sizes $\beta^{3}$ and $\beta^{4}$. In some of these situations, the LERP algorithm required a lower amount of regenerators than the lower bound. Again, this is due to the higher number of blocked lightpaths in the LERP solution, causing an overall decrease of the regeneration requirements.

Summarizing the comparison of the heuristics, the LS and 3-Step algorithms provide very similar solutions in all the occasions, very close to the optimal solutions. In Internet 2 and NSFNET cases, they were more frequent the cases in which the LS algorithm provided better solutions. Finally, some interesting remarks can be made by observing the regenerators placed by 3-Step algorithm in its second and third step. It has been observed that in practically all of the cases, no regenerators were placed in step 2 of the algorithm. That means that regenerators were seldom needed for wavelength conversion purposes. Only in some problem instances at higher loads, some lightpaths (below $0.5 \%$ ) changed their wavelength along the path. The uncommonness of the wavelength conversion in offline planned optical networks is supported by previous studies like [33].

By observing the values in the OptILP column of Table II and the results from the LS and 3-Step heuristics in Table III, it is possible to capture some trends in the regenerator equipment cost. It seems clear that irrespective of the network load, networks with a small normalized size $\left(\beta=\beta^{M I N}\right)$ do not require regeneration equipment. After that, larger network sizes are associated to higher regeneration needs. The same happens with the network load conditions, if we measure the regenerator equipment in absolute values. However, in some occasions higher load values implied lower per-lightpath regenerator cost. We observed that this behavior is explained by how the traffic matrices are synthesized. The round operation in equation (4) adds a sort of uncontrolled effect, depending on whether the coordinates of the lightpath demand matrix corresponding to distant nodes are more frequently rounded up or rounded down at a given network load. Finally, the regeneration equipment cost showed to be significantly topology-dependent. For the maximum network factor $\beta^{M A X}$, the number of regeneration units per carried lightpath was in the order of $60 \%$ for Internet 2 network, and $30 \%$ for the NSFNET and NOBEL-EU topologies. The reason for that difference can be found in the ratio between the maximum and average link lengths in the network. In Internet 2 topology, the maximum link length is only 1.6 times the size of the average link length, while in NSFNET and NOBEL-EU topologies, there is a higher disparity in link lengths. Then, the average link length measured when we normalize the network at $\beta=\beta^{M A X}$ is significantly higher in the Internet2 topology $(\sim 1800 \mathrm{~km}$ in Internet2, and $\sim 1000 \mathrm{~km}$ in NSFNET and NOBEL-EU). Consequently, the percentage of the paths that need signal regeneration at $\beta=\beta^{M A X}$ is also higher in the Internet 2 topology.

\section{Algorithms execution time}

Table II and III display the execution time observed in each of the tests performed for all the algorithms but the 3-Step heuristic. The execution times for the 3-Step algorithm are not shown since they were below of 1 second in all the tests.

Naturally, the highest execution times correspond to the exact problem solving, in OptILP column. For Internet2, the execution times were reasonable, below two minutes in all the occasions. In the NSFNET network, execution times ranged from minutes to several hours. The longest time observed was about 80 hours. Running times are higher for a higher number of nodes in the network and a higher number of wavelengths. This is caused by the increase in the number of decision variables of the problem. The effects of the network load and the normalized network size factors are more random since they affect the performance of the branch-and-bound pruning step. As general trend, longer running times were observed for low to medium normalized network size factors.

The execution of the LS algorithm has shown to be around 1 second in all the Internet 2 tests, and for NSFNET $W=8$. The rest of the execution times are in the order of tens of seconds, always below 2 minutes. Longer execution times seem to be associated to larger networks with normalized size factors $\beta^{3}$ and $\beta^{4}$. However, this trend is not deterministic, since it is again affected by the performance of the branchand-bound algorithm solving the LS formulation. Finally, the response time of the LERP algorithm is in general longer than the 3-Step and LS execution times.

\section{CONCLUSIONS}

This paper investigates the offline network planning and regenerator placement in translucent optical networks, minimizing the regenerator placement cost. As far as the authors know, we provide the first ILP model to optimally solve the problem. Thanks to its simplicity, it is able to solve problem instances in small to medium scale networks. For 
larger network topologies, we present two heuristic algorithms named LS and 3-Step. An extensive battery of tests is conducted, ranging different network topologies, number of wavelengths per fiber, traffic load conditions and network link lengths. The traffic load in the tests is normalized to fairly assess the ability of the algorithms for minimizing the regeneration cost, without producing any signal-regeneration related lightpath blocking. The network link lengths in the tests are normalized to observe the performance of the algorithms ranging different networks sizes. The results show that the LS and 3-Step algorithms provide optimal or close-tooptimal solutions in all these tests. They outperform previous heuristic algorithm presented, both in the quality of the solution found and the algorithm execution time. In addition, the 3-Step algorithm guarantees that no signal regeneration lightpath blocking is produced. Consequently, both algorithms can be used to efficiently solve the IA-RWA-RP problem (e.g. selecting the best solution provided by both schemes). Our results also show that regenerator equipment is seldom required for wavelength conversion purposes, and just needed for pure signal regeneration.

\section{REFERENCES}

[1] J. Berthold, A. A. M. Saleh, L. Blair, and J. M. Simmons, "Optical Networking: Past, Present, and Future," in IEEE Journal of Lightwave Technology, vol. 26, no. 9, pp.1104-1118, May 2008.

[2] B. Ramamurthy, D. Datta, H. Feng, J. P. Heritage, and B. Mukherjee, "Transparent vs. opaque vs. translucent wavelength-routed optical networks," in Proc. .OFC, 1999, pp. 59-61.

[3] S. Azodolmolky, M. Klinkowski, E. Marin, D. Careglio, J. Solé Pareta, and I. Tomkos, "A Survey on Physical Layer Impairments Aware Routing and Wavelength Assignment Algorithms in Optical Networks," in Computer Networks (Elsevier), vol. 53, no. 7, pp. 926 - 944, 2009.

[4] T. Tsuritani et al., "Optical path computation element interworking with network management system for transparent mesh networks," in Proc. OFC/NFOEC 2008, San Diego, USA, Mar. 2008.

[5] P. Pavon-Mariño et al., "Offline Impairment Aware RWA Algorithms for Cross-Layer Planning of Optical Networks," OSA/IEEE J. Lightw. Technol., June 2009.

[6] A. Morea, N. Brogard, F. Leplingard, J.C. Antona, T. Zami, B. Lavigne, and D. Bayart, "QoT function and A* routing an optimized combination for connection search in translucent networks," J. Optical Netw., vol. 7, no. 1, pp. 42-61, 2008.

[7] M. Yannuzzi, M. Quagliotti, G. Maier, E. Marin-Tordera, X. MasipBruin, S. Sanchez-Lopez, J. Sole-Pareta, W. Erangoli, and G. Tamiri, "'Performance of translucent optical networks under dynamic traffic and uncertain physical-layer information," in Proc. of the 13th IFIP/IEEE Conference on Optical Network Design and Modelling (ONDM 2009), Braunschweig, Germany, Feb. 2009.

[8] A. A. M. Saleh, "Islands of transparency - an emerging reality in multiwave optical networking," in 11th Annual Meeting IEEE Lasers and Electro-Optics Society, New York, USA, 1998, p. 36.

[9] A. M. Saleh, "Transparent optical networking in backbone networks," in Proc. OFC, Baltimore, USA, 2000, pp. 62-64.

[10] X. Yang and B. Ramamurthy, "Dynamic routing in translucent WDM optical networks: The intra-domain case," IEEE/OSA J. Lightw. Technol., vol. 23, no. 3, pp. 955-971, 2005.

[11] S.-W. Kim, S.-W. Seo, and S. C. Kim, "Regenerator placement algorithms for connection establishment in all-optical networks," in Proc. Golbecom 2000, vol. 2, Nov. 2000, pp. 1205-1209.
[12] B. Ramamurthy, S. Yaragorla, and X. Yang, "Translucent optical WDM networks for the next-generation backbone networks," in Proc.Globecom 2001, Texas, USA, Dec. 2001, pp. 60-64.

[13] X. Yang and B. Ramamurthy, "Sparse regeneration in a translucent WDM optical network," in Proc. Asia Pacific Optical and Wireless Communication Conference (APOC), Beijing, China, 2001, pp. 61-70.

[14] M. A. Ezzahdi, S. A. Zahr, M. Koubaa, N. Puech, and M. Gagnaire, "LERP: A Quality of transmission dependent heuristic for routing and wavelength assignment in hybrid WDM networks," in Proc. ICCCN 2006, Virginia, USA, 2006, pp. 125-136.

[15] Y. Ye, T. Y. Chai, T. H. Cheng, and C. Lu, "Algorithms for the design of WDM translucent optical networks," Opt. Express, vol. 11, no. 22, pp. 2917-2926, 2003.

[16] S. Al Zahr, M. Gagnaire, N. Puech, "Impact of wavelength assignment strategies on hybrid WDM network planning", in Proc. of 6th International Workshop on Design and Reliable Communication Networks (DRCN2007), La Rochelle, France, 2007.

[17] S. Al Zahr, N. Puech, and M. Gagnaire, "Gain equalization versus electrical regeneration tradeoffs in hybrid WDM networks," in Proc. 9th International Conference on Telecommunications (ConTEL), Zagreb, Croatia, 2007.

[18] K. Manousakis, K. Christodoulopoulos, E. Kamitsas, I. Tomkos, and E.A. Varvarigos, "Offline impairment-aware routing and wavelength assignment algorithms in translucent WDM optical networks," IEEE/OSA J. Lightw. Technol., vol. 27, no. 12, pp. 1866-1877, Jun. 2009.

[19] S. Pachnicke, T. Paschenda, P.M. Krummrich, "Physical impairment based regenerator placement and routing in translucent optical networks," in Proceedings of OFC/NFOEC 2008, San Diego, CA, USA, Feb. 2008.

[20] G. Shen, W. D. Grover, T. Hiang Cheng, and S. K. Bose, "Sparse placement of electronic switching nodes for low blocking in translucent optical networks," Journal of Optical Networking, vol. 1, Issue 12, p.424-441, Dec. 2002.

[21] X. Yang, B. Ramamurthy, "Sparse regeneration in translucent wavelength-routed optical networks: architecture, network design and wavelength routing," Photonic Network Communications, vol. 10, no. 1, pp. 39-50, 2005.

[22] M. S. Savasini, P. Monti, M. Tacca, A. Fumagalli, and H. Waldman, "Regenerator placement with guaranteed connectivity in optical networks," in Proc. of the IEEE ONDM conference, Athens, Greece, Jun. 2007, pp. 438-447.

[23] S. Pachnicke, T. Paschenda, P. Krummrich, "Assessment of a constraint based routing algorithm for translucent 10 Gbits/s DWDM networks considering fiber nonlinearities," Journal of Optical Networking, vol. 7, Issue 4, pp. 365-377, 2008.

[24] S. Chen and S. Raghavan, "The regenerator location problem," Working paper, Smith School of Business, University of Maryland, 2006.

[25] S. D. Personick, "Receiver Design for Digital Fiber Optic Communication Systems, I," Bell Syst. Tech. J, vol. 52, no. 6, pp. 843874, July-August 1973.

[26] R. Ramaswami and K. N. Sivarajan, "Routing and Wavelength Assignment in All-Optical Networks," IEEE/ACM Transactions on Networking, vol. 3, no. 5, pp. 489-500, Oct. 1995.

[27] L. Kleinrock, Queueing systems: Computer applications, vol. 2, Wiley, New York, 1976.

[28] P. Pavon-Marino, R. Aparicio-Pardo, G. Moreno-Muñoz, J. GarciaHaro, and J. Veiga-Gontan, "MatPlanWDM: An educational tool for network planning in wavelength-routing networks," in Proceedings of IFIP Int. Conf. Optical Network Design and Modeling, Athens, Greece, 2007, pp. 58-67.

[29] K. Holmström, A. O. Göran, and M. M.Edvall, "User's Guide for TOMLAB/CPLEX v11.2", 2009.

[30] Internet 2 Global Research Network Operations Center, Web Site, [Online], Available: http://www.abilene.iu.edu/.

[31] R. Ramaswami, K.N. Sivarajan, "Design of Logical Topologies for Wavelength-routed Optical Networks," IEEE Journal on Selected Areas in Communications, vol. 14, no. 5, pp. 840-851, Jun. 1996. 
[32] SNDlib 1.0-Survivable network design data library. http://sndlib.zib.de, 2005.

[33] P. Pavon-Marino, R. Aparicio-Pardo, B. Garcia-Manrubia, J. FernandezPalacios, O. Gonzalez, F. Martin, and J. Garcia-Haro, "Balancing multifibre and wavelength converter cost in wavelength routing networks," in Proc. 34th European Conference on Optical

Communication, ECOC 2008, vol. 5, Brussels, Belgium, Sept. 2008, pp. 185-186.

TABLE II

INTERNET2 AND NSFNET RESULTS

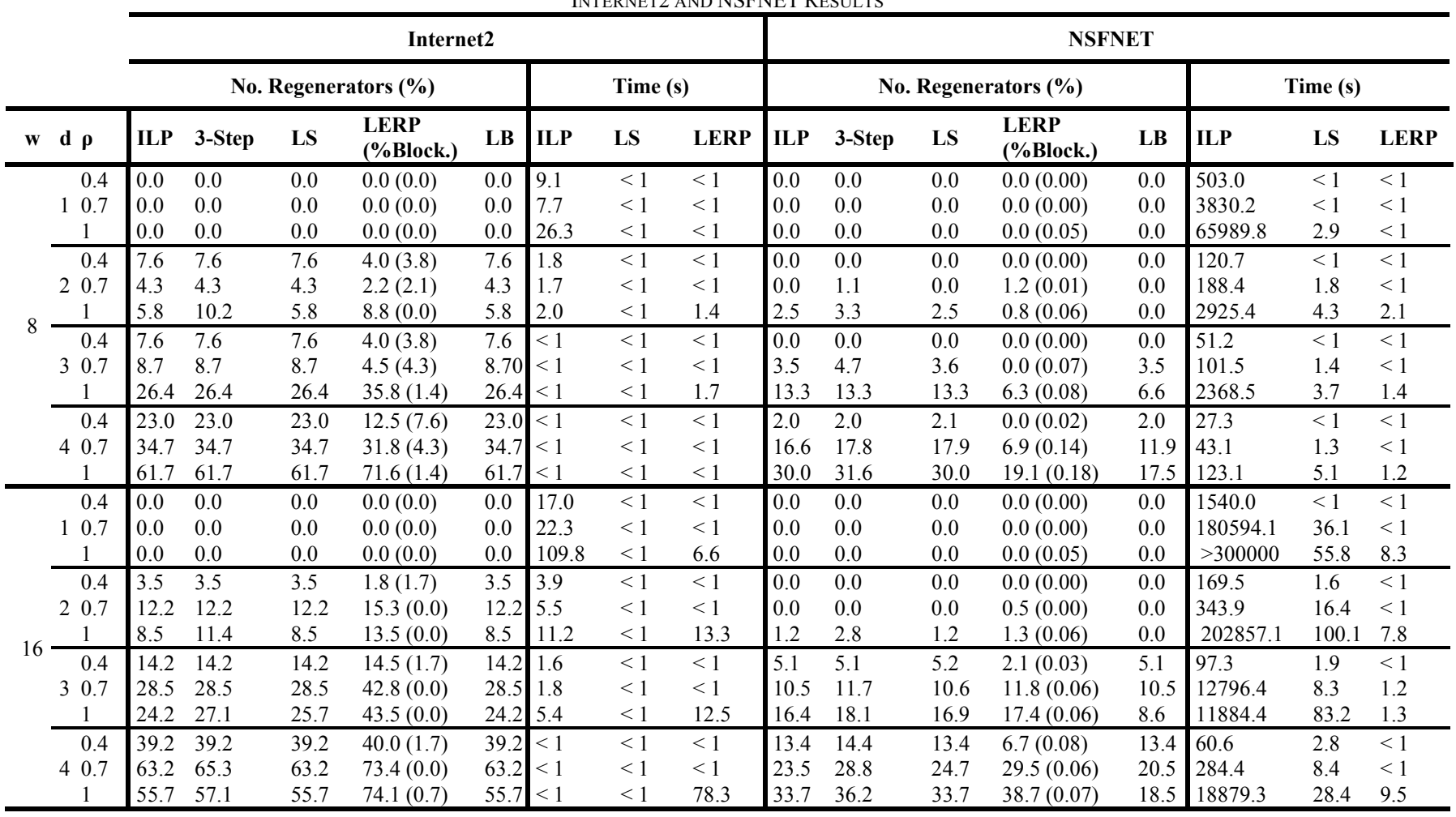

TABLE III

NOBEL-EU RESULTS

\begin{tabular}{cl|llll|lc}
\multicolumn{7}{c|}{} & \multicolumn{3}{c|}{ No. Regenerators (\%) } & \multicolumn{2}{c}{ Time (s) } \\
\hline \multirow{2}{*}{ d } & $\boldsymbol{\rho}$ & 3-Step & LS & $\begin{array}{l}\text { LERP } \\
\text { (\%Block.) }\end{array}$ & LB & LS & LERP \\
\hline & 0.4 & 0.0 & 0.0 & $0.0(0.0)$ & 0.0 & 3.7 & 23.4 \\
1 & 0.7 & 0.0 & 0.0 & $0.0(0.0)$ & 0.0 & 4.8 & 43.2 \\
& 1 & 0.0 & 0.0 & $0.0(0.0)$ & 0.0 & 7.4 & 61.0 \\
\hline & 0.4 & 0.0 & 0.0 & $0.0(0.0)$ & 0.0 & 4.9 & 23.4 \\
2 & 0.7 & 0.0 & 0.0 & $0.0(0.0)$ & 0.0 & 5.9 & 43.0 \\
& 1 & 0.0 & 0.0 & $0.0(0.0)$ & 0.0 & 8.2 & 61.0 \\
\hline & 0.4 & 5.6 & 5.6 & $2.2(3.5)$ & 5.6 & 11.3 & 23.5 \\
3 & 0.7 & 8.5 & 8.5 & $7.4(2.4)$ & 8.5 & 15.7 & 43.1 \\
& 1 & 7.0 & 7.0 & $6.1(1.8)$ & 7.0 & 18.0 & 60.8 \\
\hline & 0.4 & 18.4 & 18.4 & $10.5(8.8)$ & 18.4 & 12.4 & 23.5 \\
4 & 0.7 & 29.1 & 29.1 & $31.1(7.6)$ & 29.1 & 27.0 & 43.2 \\
& 1 & 24.7 & 24.7 & $26.3(6.3)$ & 24.7 & 60.0 & 61.0 \\
\hline
\end{tabular}

